\title{
Optimized BER Performance of Asymmetric Turbo Codes over AWGN Channel
}

\author{
M.Srinivasa Rao \\ Pvpsit, JNTU Kakinada \\ Vijayawada \\ Andhra Pradesh, India.
}

\author{
G.Vijaya Kumar \\ Pvpsit, JNTU Kakinada \\ Vijayawada \\ Andhra Pradesh, India.
}

\author{
P.Rajesh Kumar, Ph.D \\ Pvpsit, JNTU Kakinada \\ Vijayawada \\ Andhra Pradesh, India.
}

\begin{abstract}
The performance of the turbo codes can be improved by varying parameters such as block size, code polynomial structure and constraint length. This paper proposes a new asymmetric turbo codes that consists of parallel concatenated component codes with mixed type generator polynomials and also different constraint lengths. This scheme optimizes the BER performance of asymmetric turbo codes of both Waterfall region at lower SNR's and error-floor region at higher SNR's. The simulation results shows better BER performance of asymmetric turbo codes for block size of 128, 512 bits, code-rate of 1/2 and Maximum-A-Posteriori (MAP) decoding algorithm over AWGN channel with BPSK modulation by using MATLAB.
\end{abstract}

\section{Keywords}

Asymmetric turbo codes, bit error rate, constraint length, code-rate, error-floor and waterfall.

\section{INTRODUCTION}

In 1993, turbo codes are introduced by Berrou, Glavieux and Thitimajshima. These codes are introduced as one of the most powerful error control codes which were the first practical codes to closely approach the channel capacity. They are basically constructed by two or more parallel Recursive Systematic Convolutinal (RSC) codes, which are linked by a pseudo-random interleaver [1][3].

The bit error rate (BER) performance curve of turbo codes can be divided into "waterfall" region and "error-floor" region. The "waterfall" region has a steep slope at lower SNR's for a long block of information and "error-floor" region has a shallower slope at higher SNR's caused by codeword of small weight [2].

The turbo codes can be divided into two types based on their generator polynomial structures and constraint lengths. The turbo codes basically use the identical component codes known as symmetric turbo codes which have same constraint length and generator polynomials [1]. The symmetric turbo codes have either a good "waterfall" BER performance or a good "error floor" BER performance, but not both. The parallel concatenated codes which uses non-identical component codes known as asymmetric turbo codes. It has not only different constraint lengths but also different generator polynomials [8]. The resulting asymmetric turbo code has both good "waterfall" and "error-floor" BER performance [2][7].

The decoding of a turbo codes can be done in an iterative way using Maximum a posteriori (MAP) algorithm [1]. In this paper, we consider BPSK (Binary Phase Shift Keying) modulation over AWGN channel. This paper proposes the new asymmetric turbo codes, which consists of a parallel concatenated turbo codes that uses the mixed type of the component codes with different not only the constraint length but also the generator polynomial. We can observe the optimized BER performance in the both "waterfall" region and the "error floor" region by applying the proposed asymmetric turbo codes.

\section{ASYMMETRIC TURBO CODES}

\subsection{Asymmetric Turbo Encoder}

A turbo encoder is constructed by a parallel concatenation of two identical component codes, which are linked by a pseudorandom interleaver. Fig.1 shows a turbo encoder (rate 1/2) structure with two RSC encoders. Here trellis termination or truncation is performed on RSC encoders.

Trellis termination is performed on the first RSC encoder, which returns its memory contents to zero state, while trellis truncation is performed on the second RSC encoder that leaves its memory states open.

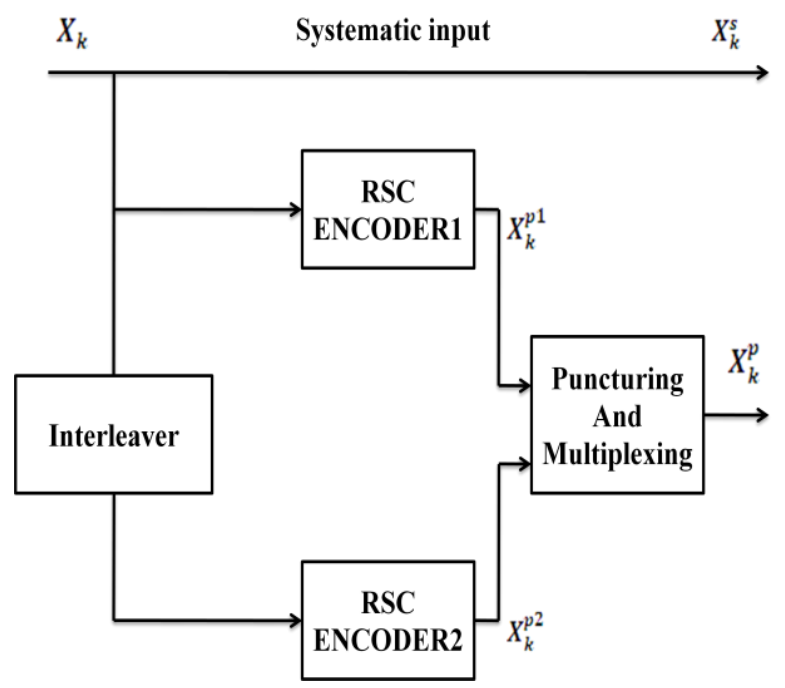

Fig.1. Generic Turbo Encoder

Puncturing is a technique used to increase the code rate. A rate $1 / 3$ encoder is converted to a rate $1 / 2$ encoder by multiplexing the two coded streams. The multiplexer can choose the odd indexed outputs from the output of the upper RSC encoder and its even indexed outputs from the lower one. 


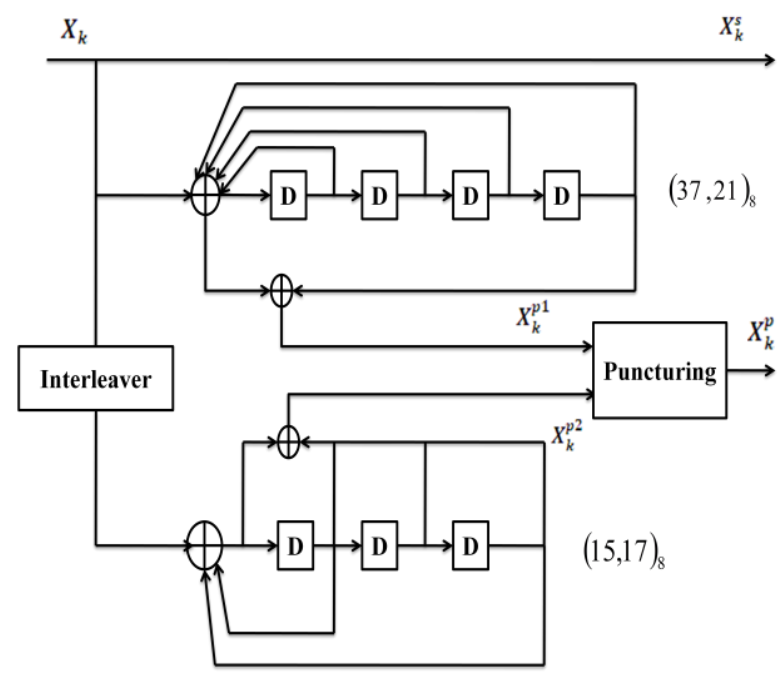

Fig.2 Block Diagram of asymmetric turbo encoder with $K 1=5, K 2=4$ and $R=1 / 2$

Fig. 2 shows the asymmetric turbo encoder with the code rate $1 / 2$, which consists of $(37,21)_{8}$ encoder and $(15,17)_{8}$ encoder, Where $\mathrm{K} 1$ and $\mathrm{K} 2$ are constraint lengths of turbo encoder 1 and turbo encoder 2 , respectively and $\mathrm{R}$ is the code rate for the turbo codes

\subsection{Asymmetric Turbo Decoder}

In a typical turbo decoding system (see Fig. 3), two decoders operate iteratively and pass their decisions to each other after each iteration [4].

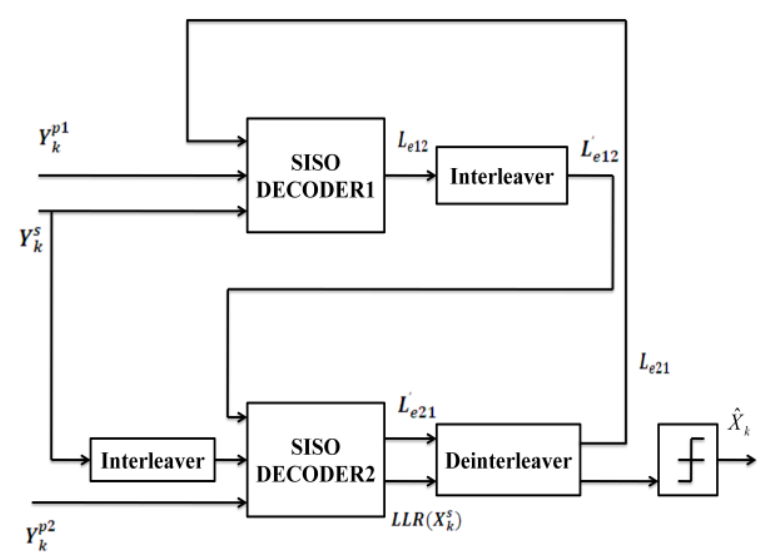

Fig.3. Iterative Turbo Decoding

Each decoder operates not only on its input but also on the other decoder's incompletely decoded output. Here, Encoded information sequence $X_{k}$ is transmitted over an AWGN channel, and a noisy received sequence $Y_{k}$ is obtained. Every SISO decoder determines the Log-Likelihood Ratio (LLR) for the kith data bit $d_{k}$ as:

$$
L\left(d_{k}\right)=\log \left[\frac{P\left(d_{k}=1 \mid Y\right)}{P\left(d_{k}=0 \mid Y\right)}\right]
$$

LLR can be composed into 3 independent terms as:

$$
L\left(d_{k}\right)=L_{a p r i}\left(d_{k}\right)+L_{c}\left(d_{k}\right)+L_{e}\left(d_{k}\right)
$$

Where $L_{a p r i}\left(d_{k}\right)$ is the a-priori information, $L_{c}\left(d_{k}\right)$ is the channel measurement, and $L_{e}\left(d_{k}\right)$ is the extrinsic information exchanged between the component decoders. The available extrinsic information from one decoder will be a-priori information for the other decoder at the next decoding stage. Where $L_{e 12}$ and $L_{e 21}$ in Fig.3 represent the extrinsic information from decoder 1 to decoder2 and decoder2 to decoder1 respectively [5][6].

MAP algorithm calculates the LLRs for each information bit as:

$$
L\left(d_{k}\right)=\ln \left[\frac{\sum_{S_{k}} \sum_{S_{k-1}} \gamma_{1}\left(S_{k-1}, S_{k}\right) \alpha\left(S_{k-1}\right) \beta\left(S_{k}\right)}{\sum_{S_{k}} \sum_{S_{k-1}} \gamma_{0}\left(S_{k-1}, S_{k}\right) \alpha\left(S_{k-1}\right) \beta\left(S_{k}\right)}\right]
$$

Where $\alpha$ is the forward recursion, $\beta$ is the backward recursion, $\gamma$ is the branch metric, and $S_{k}$ is the trellis time k. Forward recursion are calculated from trellis time $\mathrm{K}=1$ to, $\mathrm{K}=\mathrm{N}$ where $\mathrm{N}$ is the number of information bits in one data frame. Recursive calculation of forward state metrics is performed as:

$$
\alpha_{k}\left(S_{k}\right)=\sum_{j=0}^{1} \alpha_{k-1}\left(S_{k-1}\right) \gamma_{j}\left(S_{k-1}, S_{k}\right)
$$

Similarly, the backward recursions are calculated from trellis time $\mathrm{K}=\mathrm{N}$ to, $\mathrm{K}=1$ as:

$$
\beta_{k}\left(S_{k}\right)=\sum_{j=0}^{1} \beta_{k+1}\left(S_{k+1}\right) \gamma_{j}\left(S_{k}, S_{k+1}\right)
$$

Branch metrics are calculated for each possible trellis transition as:

$$
\begin{aligned}
& \gamma_{i}\left(S_{k-1}, S_{k}\right)=C_{k} P\left(S_{k} \mid S_{k-1}\right) \times \\
& \exp \left[\frac{2}{N_{0}}\left(Y_{k}^{s} X_{k}^{s}(i)+Y_{k}^{p} X_{k}^{p}\left(i, S_{k-1}, S_{k}\right)\right)\right]
\end{aligned}
$$

Where $i=(0,1), C_{k}$ is a constant, $X_{k}^{s}$ and $X_{k}^{p}$ are the encoded systematic data bit and parity bit, and $Y_{k}^{s}$ and $Y_{k}^{p}$ are the received noisy systematic data bit and parity bit respectively.

\section{PRPOSED ASYMMETRIC TURBO CODES}

The proposed asymmetric turbo codes that uses parallel concatenated component encoders with mixed type of generator polynomials and different constraint lengths. Turbo codes with larger constraint length will achieve good performance and have better free distance but the computational complexity increases. It is necessary to decrease the constraint length of one of the component encoder in order to obtain good BER performance and reduce decoding delay.

Table. 1 shows different types of asymmetric turbo codes with different constraint length and generator polynomials. Here $\mathrm{P}$ 
and NP describe primitive and prime polynomial for component encoders. $\mathrm{G}$ and $\mathrm{K}$ represent the feedback generator polynomial and the constraint length of each component codes, respectively. There are four types of turbo codes from each component codes: $(\mathrm{NP}, \mathrm{P})$ turbo codes, $(\mathrm{P}$, $\mathrm{NP})$ turbo codes, $(\mathrm{P}, \mathrm{P})$ turbo codes, $(\mathrm{NP}, \mathrm{NP})$ turbo codes. Here $(\mathrm{P}, \mathrm{P})$ turbo codes uses primitive polynomial for the feedback polynomial of all the component codes, and (NP, NP) turbo codes uses prime polynomial for the feedback polynomial of both the component codes but performance is similar to the conventional turbo codes. (P, NP) and (NP, P) uses primitive and prime (non-primitive) polynomial for the feedback polynomial of each component codes.

Table1. Different types of asymmetric turbo codes with the different constraint lengths

\begin{tabular}{|c|c|c|c|c|}
\hline $\mathrm{G}$ & $\begin{array}{c}\text { (P,NP) } \\
\text { Turbo codes }\end{array}$ & $\begin{array}{c}\text { (NP,P) } \\
\text { Turbo codes }\end{array}$ & $\begin{array}{c}\text { (NP,NP) } \\
\text { Turbo codes }\end{array}$ & $\begin{array}{c}(\mathrm{P}, \mathrm{P}) \\
\text { Turbo codes }\end{array}$ \\
\hline $\begin{array}{l}\mathrm{Kl}=3, \\
\mathrm{~K} 2=3\end{array}$ & $\begin{array}{l}\mathrm{Gl}=(7,5), \\
\mathrm{G} 2=(5,5)\end{array}$ & $\begin{array}{l}\mathrm{Gl}=(\mathbf{5 , 5 )}, \\
\mathrm{G} 2=(7,5)\end{array}$ & $\begin{array}{l}\mathrm{Gl}=(5,5), \\
\mathrm{G} 2=(5,5)\end{array}$ & $\begin{array}{l}\mathrm{Gl}=(7,5), \\
\mathrm{G} 2=(7,5)\end{array}$ \\
\hline $\begin{array}{l}\mathrm{K} l=3, \\
\mathrm{~K} 2=4\end{array}$ & $\begin{array}{l}\mathrm{Gl}=(7,5), \\
\mathrm{G} 2=(15,17)\end{array}$ & $\begin{array}{l}\mathrm{Gl}=(\mathbf{5 , 5}), \\
\mathrm{G} 2=(15,17)\end{array}$ & $\begin{array}{l}\mathrm{Gl}=(5,5), \\
\mathrm{G} 2=(17,17)\end{array}$ & $\begin{array}{l}\mathrm{Gl}=(7,5), \\
\mathrm{G} 2=(15,17)\end{array}$ \\
\hline $\begin{array}{l}\mathrm{Kl}=3, \\
\mathrm{~K} 2=5\end{array}$ & $\begin{array}{l}\mathrm{Gl}=(7,5), \\
\mathrm{G} 2=(37,21)\end{array}$ & $\begin{array}{l}\mathrm{Gl}=(5,5), \\
\mathrm{G} 2=(23,25)\end{array}$ & $\begin{array}{l}\mathrm{Gl}=(5,5), \\
\mathrm{G} 2=(37,2 \mathrm{l})\end{array}$ & $\begin{array}{l}\mathrm{Gl}=(7,5), \\
\mathrm{G} 2=(23,35)\end{array}$ \\
\hline $\begin{array}{l}\mathrm{K} l=4, \\
\mathrm{~K} 2=3\end{array}$ & $\begin{array}{l}\mathrm{Gl}=(15,17), \\
\mathrm{G} 2=(5,5)\end{array}$ & $\begin{array}{l}\mathrm{Gl}=(17,17), \\
\mathrm{G} 2=(7,5)\end{array}$ & $\begin{array}{l}\mathrm{Gl}=(17,17), \\
\mathrm{G} 2=(5,5)\end{array}$ & $\begin{array}{l}\mathrm{Gl}=(15,17, \\
\mathrm{G} 2=(7,5)\end{array}$ \\
\hline $\begin{array}{l}\mathrm{Kl}=4, \\
\mathrm{~K} 2=4\end{array}$ & $\begin{array}{l}\mathrm{Gl}=(15,17), \\
\mathrm{G} 2=(17,17)\end{array}$ & $\begin{array}{l}\mathrm{Gl}=(17,17), \\
\mathrm{G} 2=(15,17)\end{array}$ & $\begin{array}{l}\mathrm{Gl}=(17,17), \\
\mathrm{G} 2=(17,17)\end{array}$ & $\begin{array}{l}\mathrm{Gl}=(15,17), \\
\mathrm{G} 2=(15,17)\end{array}$ \\
\hline $\begin{array}{l}\mathrm{Kl}=4, \\
\mathrm{~K} 2=5\end{array}$ & $\begin{array}{l}\mathrm{Gl}=(15,17), \\
\mathrm{G} 2=(37,21)\end{array}$ & $\begin{array}{l}\mathrm{Gl}=(17,17), \\
\mathrm{G} 2=(23,35)\end{array}$ & $\begin{array}{l}\mathrm{Gl}=(17,17), \\
\mathrm{G} 2=(37,21)\end{array}$ & $\begin{array}{l}\mathrm{Gl}=(15,17), \\
\mathrm{G} 2=(23,35)\end{array}$ \\
\hline $\begin{array}{l}\mathrm{Kl}=5, \\
\mathrm{~K} 2=3\end{array}$ & $\begin{array}{l}\mathrm{Gl}=(23,35), \\
\mathrm{G} 2=(5,5)\end{array}$ & $\begin{array}{l}\mathrm{Gl}=(37,2 \mathrm{l}), \\
\mathrm{G} 2=(7,5)\end{array}$ & $\begin{array}{l}\mathrm{Gl}=(37,2 \mathrm{l}), \\
\mathrm{G} 2=(5,5)\end{array}$ & $\begin{array}{l}\mathrm{Gl}=(23,35), \\
\mathrm{G} 2=(7,5)\end{array}$ \\
\hline $\begin{array}{l}\mathrm{Kl}=5, \\
\mathrm{~K} 2=4\end{array}$ & $\begin{array}{l}\mathrm{Gl}=(23,35), \\
\mathrm{G} 2=(17,17)\end{array}$ & $\begin{array}{l}\mathrm{Gl}=(37,21), \\
\mathrm{G} 2=(15,17)\end{array}$ & $\begin{array}{l}\mathrm{Gl}=(37,21), \\
\mathrm{G} 2=(17,17)\end{array}$ & $\begin{array}{l}\mathrm{Gl}=(23,35), \\
\mathrm{G} 2=(15,17)\end{array}$ \\
\hline $\begin{array}{l}\mathrm{K} l=5, \\
\mathrm{~K} 2=5\end{array}$ & $\begin{array}{l}\mathrm{Gl}=(23,35), \\
\mathrm{G} 2=(37,21)\end{array}$ & $\begin{array}{l}\mathrm{Gl}=(37,21), \\
\mathrm{G} 2=(23,35)\end{array}$ & $\begin{array}{l}\mathrm{Gl}=(37,21), \\
\mathrm{G} 2=(37,21)\end{array}$ & $\begin{array}{l}\mathrm{Gl}=(23,35), \\
\mathrm{G} 2=(23,35)\end{array}$ \\
\hline
\end{tabular}

If we use primitive polynomials as one of the feedback generator polynomials of the component encoders which shows degrading waterfall performance at lower SNR's and lower error-floor at higher SNR's. If we use non-primitive (prime) polynomials as one of the feedback generator polynomials which shows better waterfall performance at lower SNR's. So, proposed asymmetric turbo code is a combination of primitive and prime generator polynomials. The component encodes with primitive generator polynomials may increase the free distance in order to reduce error-floor and component encoders with prime generator polynomials may optimize the distance spectrum to improve water-fall performance [2].

\section{SIMULATION RESULTS}

In this paper, several combinations of asymmetric turbo codes have been studied in order to determine their performances. All simulations were performed for asymmetric turbo codes with code rate of $1 / 2$ over AWGN channel, with a BPSK modulation, block sizes of 128,512 and constraint lengths $\mathrm{K}$ are 3,4 and 5 .

Fig.4 show the BER performance curve for mixed type of asymmetric turbo codes with the same constraint lengths $\mathrm{K} 1=3, \mathrm{~K} 2=3$ and block size of 128 and 512. The simulation results of fig.4 (a) and fig.4 (b) show that both primitive type turbo codes have better the BER performance in "waterfall region" at low SNR's and "error-floor" at high SNR's compared to the other turbo codes. Here in fig.4 (NP, P) turbo codes BER performance is superior to $(\mathrm{P}, \mathrm{NP}),(\mathrm{NP}, \mathrm{NP})$ turbo codes and inferior to primitive type i.e., $(\mathrm{P}, \mathrm{P})$ turbo codes performance.

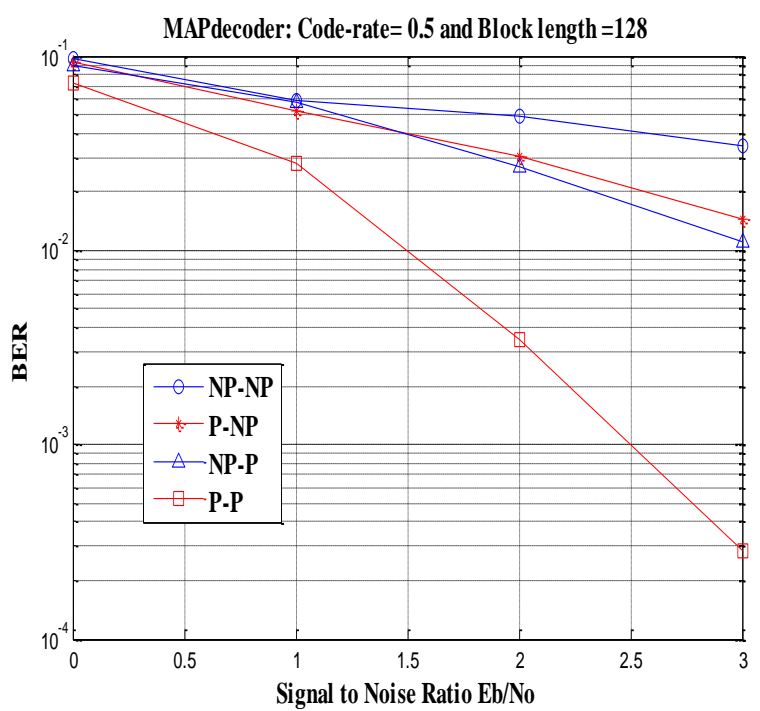

(a)

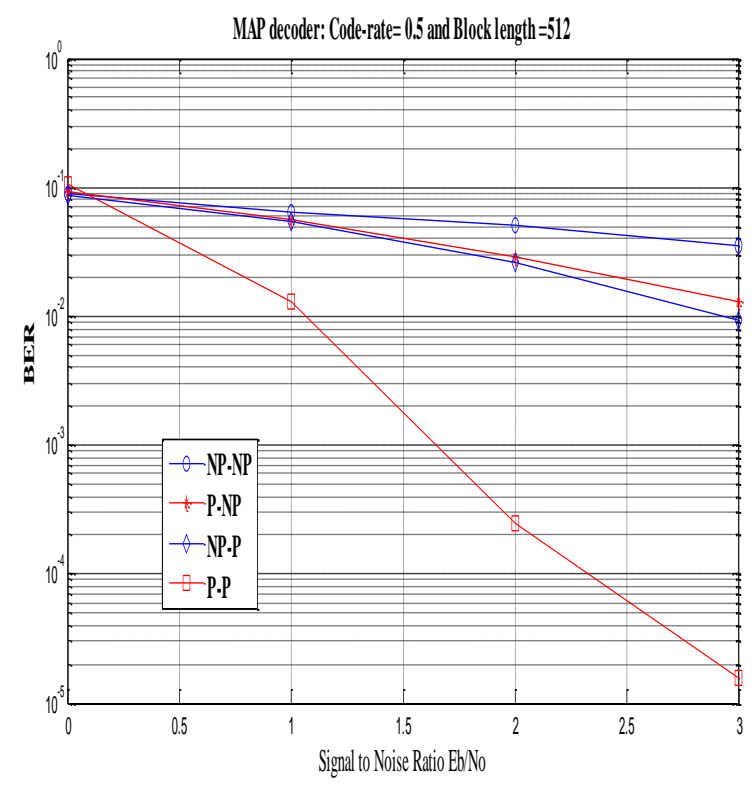

(b)

Fig.4 BER performance curve for the block size of 128 and $512(K 1=3, K 2=3)$

Fig.5 show the BER performance curve for the four types of asymmetric turbo codes with diff. constraint lengths $\mathrm{K} 1=5$, $\mathrm{K} 2=3$ and with the block size of 128,512 . The simulation results of fig.5 (a) and 5(b) show that (NP, P) and (P, P) turbo codes have better the BER performance in both "waterfall" and "error-floor" region. The BER performance of (NP, NP) and $(\mathrm{P}, \mathrm{NP})$ turbo codes are inferior to $(\mathrm{NP}, \mathrm{P})$ and $(\mathrm{P}, \mathrm{P})$ turbo codes. So, it is the best method to use one component encoder as primitive feedback generator polynomial and other as non-primitive polynomial for asymmetric turbo codes. 


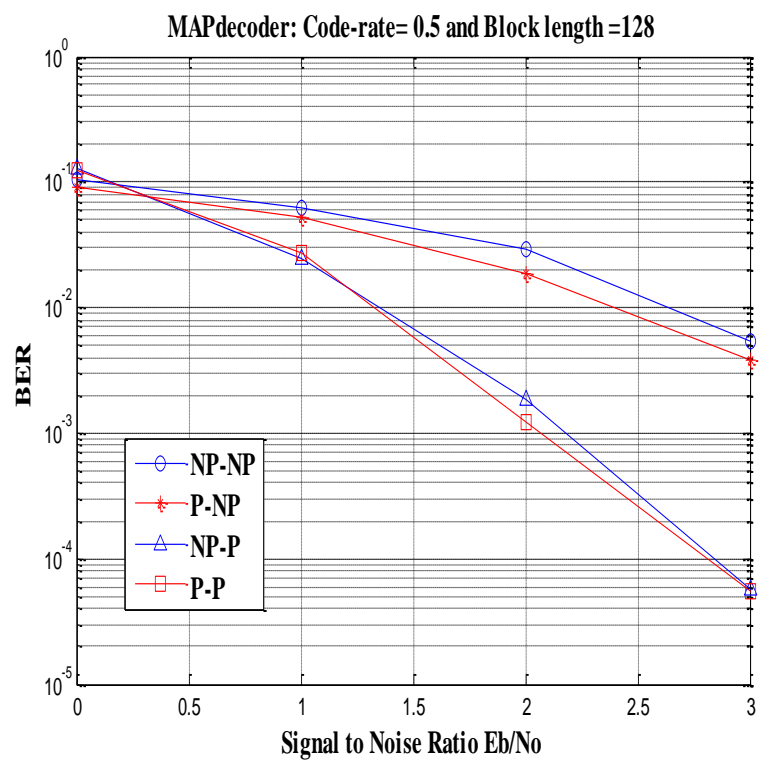

(a)

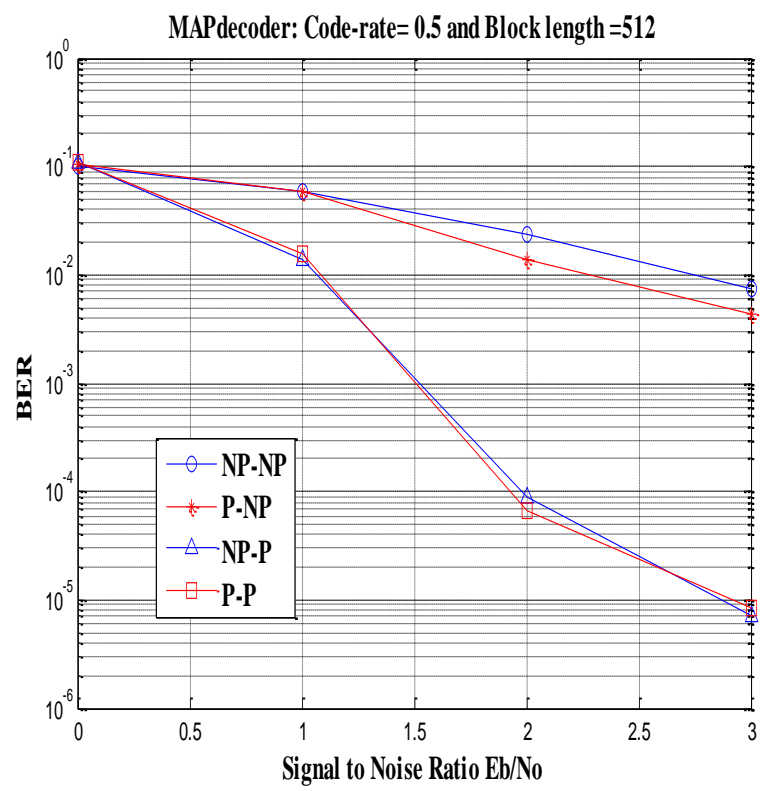

(b)

Fig.5 BER performance curve for the block size of 128 and $512(K 1=5, K 2=3)$

Fig. 6 shows the BER performance of asymmetric turbo codes with different combination of constraint length $\mathrm{K}=3$, 4 for the block size of 128 and 512. The simulation results of fig.6 (a) and $6(\mathrm{~b})$ show that BER performance of symmetric turbo codes with $\mathrm{K} 1=\mathrm{K} 2=4$ is slightly superior to asymmetric turbo codes with $\mathrm{K} 1=3, \mathrm{~K} 2=4$ or $\mathrm{k} 1=4, \mathrm{~K} 2=3$. The performance of asymmetric turbo codes with $\mathrm{K} 1=4, \mathrm{~K} 2=3$ is very close to symmetric turbo codes with constraint length $\mathrm{K} 1=\mathrm{K} 2=4$. By decreasing the constraint length of second component encoder shows better BER performance when compared with asymmetric turbo codes with different constraint lengths.

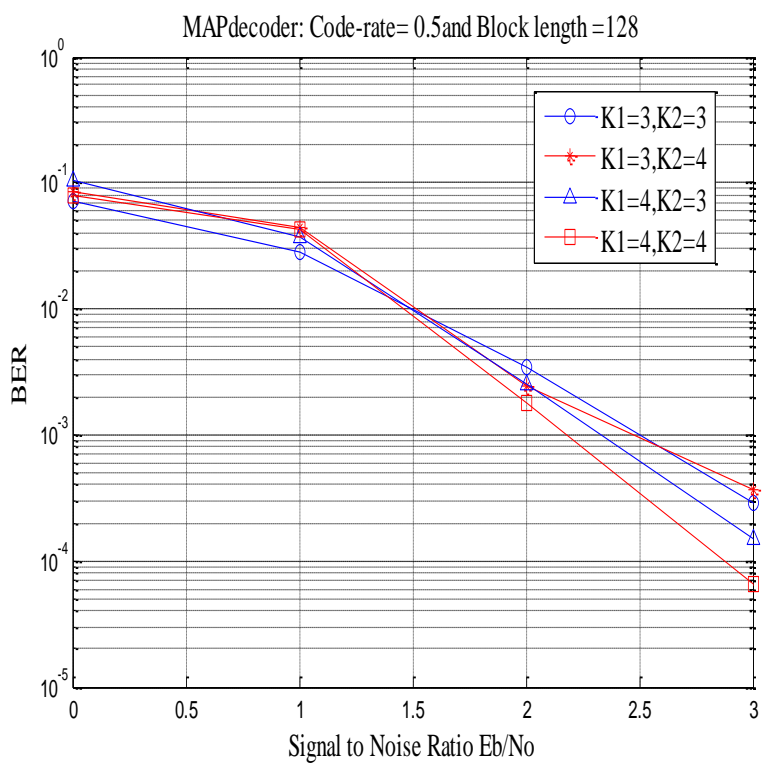

(a)

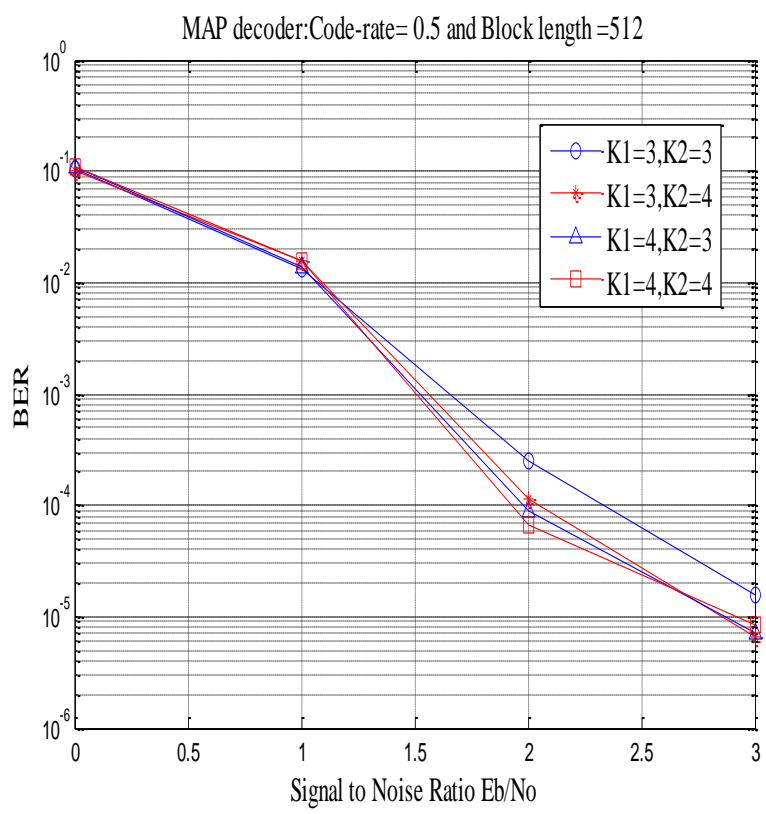

(b)

Fig.6 BER performance of $(P, P)$ turbo codes with diff. constraint lengths 3,4 for block size of 128 and 512

Fig.7 shows the BER performance both primitive type asymmetric turbo codes with different combination of constraint length $\mathrm{K}=3,4,5$ for the block size of 128 and 512 . The simulation results of fig.7 (a) and 7(b) show that performance of asymmetric turbo codes with $\mathrm{K} 1=3, \mathrm{~K} 2=5$ is inferior to turbo codes with $\mathrm{K} 1=5, \mathrm{~K} 2=3$. So, it is better to design asymmetric turbo codes with larger constraint length for first component encoder. Performance of turbo codes with $\mathrm{K} 1=\mathrm{K} 2=5$ is slightly superior to asymmetric turbo codes with $\mathrm{K} 1=4, \mathrm{~K} 2=5$ or $\mathrm{k} 1=5, \mathrm{~K} 2=4$. 


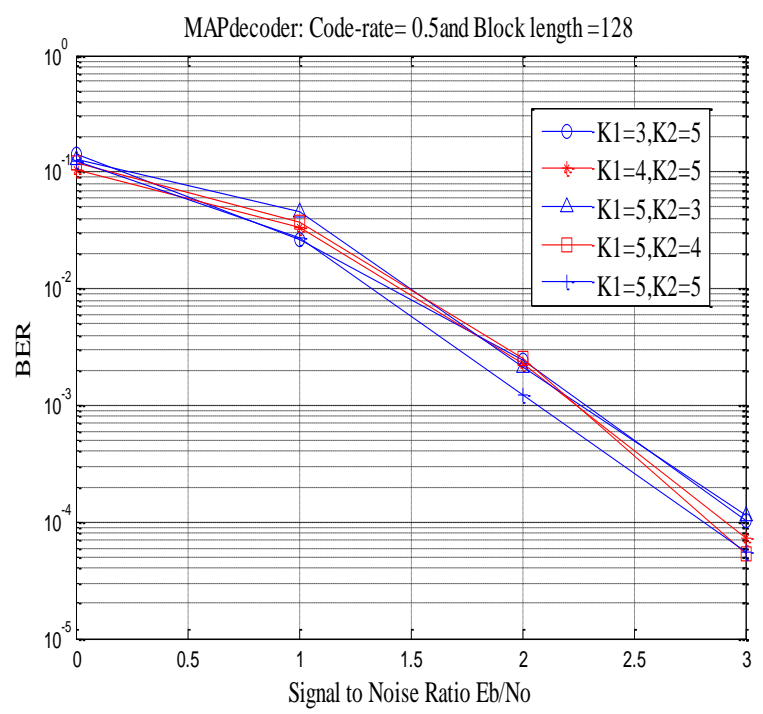

(a)

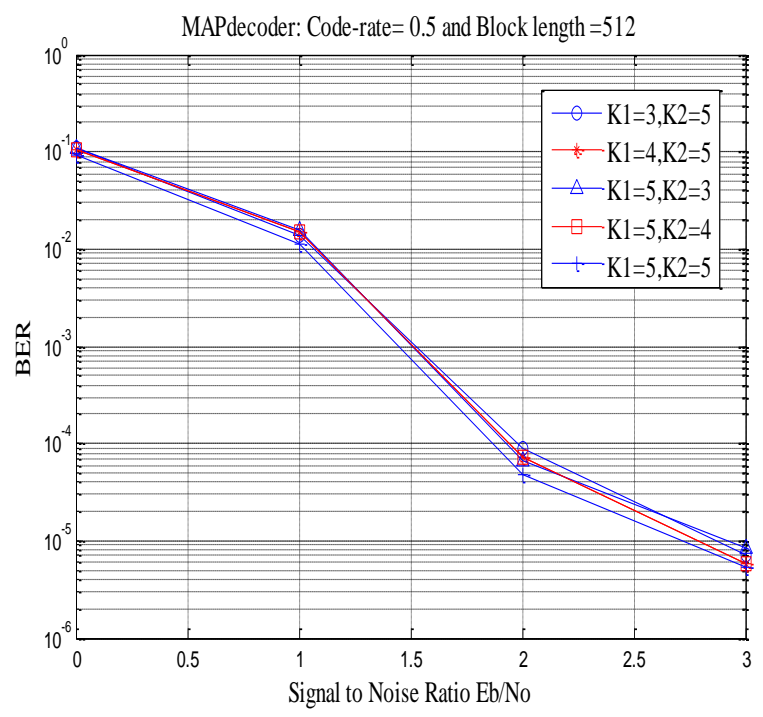

(b)

Fig.7 BER performance of $(P, P)$ turbo codes with diff. constraint lengths 3, 4, 5 for block size of 128 and 512

\section{CONCLUSION}

In this paper, the proposed asymmetric turbo codes are constructed with different constraint lengths and mixed type of generator polynomials. The proposed scheme optimizes the BER performance in both "waterfall" and "error-floor" region with the use of primitive and non-primitive polynomials alternatively. So, finally we concluded that the BER performance of asymmetric turbo codes is superior to conventional symmetric turbo codes. But the turbo codes with larger constraint length will increase delay and computational complexity. This asymmetric turbo codes widely used in mobile $3 \mathrm{G}$ communication systems.

\section{REFERENCES}

[1] C.Berrou, A.Glavieux, and P.Thitimajshima, "Near Shannon limit Error- correcting coding and decoding: Turbo codes" International Conference on Communications (ICC), pp.1064-1070, May 1993.

[2] O.Takeshita, O.M. Collins, P.Massey, and D.J. Costello, "A note on asymmetric turbo codes", in IEEE International Symposium on Information Theory (ISIT), p.179, August 1998.

[3] C.Berrou and A.Glavieux, "Near optimum error correcting coding and decoding: Turbo codes", IEEE Transactions on communications, pp. 1261-1271, October 1996.

[4] S.Pietrobon, "Implementation and performance of a turbo/MAP decoder" Int. Jour. of Satellite Communications, 1998, pp.4-17.

[5] P.Robertson, E.Villebrun, and P.Hoeher, "A comparison of optimal and sub-optimal MAP decoding algorithms operating in the log domain" in IEEE International Conference on Communications, pp.1009-1013, Seattle, USA, June 1995

[6] P.Robertson, P.Hoeher, and E.Villebrun, "Optimal and sub-optimal maximum a posteriori algorithms suitable for turbo decoding," European Trans on Telecomm. vol.8, no.2, March-April 1997, pp.119-125.

[7] K.Ramasarny, B.Balakrishna and M.Siddiqi, "A new class of asymmetric turbo codes for $3 \mathrm{G}$ systems", Int. J. Electron. Commun, June 2006, pp. 447-458.

[8] P.Massey and D.Costello, "New developments in asymmetric turbo codes", in Proc $2^{\text {nd }}$ International Symposium, Sept. 2000, pp.93-100. 